\title{
Haptic Hand-Tremor Simulation for Enhancing Empathy with Disabled Users
}

\author{
Gastone Pietro Papini Rosati, Marco Fontana, Rocco Vertechy \\ Marcello Carrozzino and Massimo Bergamasco
}

\begin{abstract}
This paper presents a system designed to induce, in healthy subjects, artificial hand-tremor that is observed in persons affected by neurological impairments. The objective is to allow a healthy user to feel in first person the effect of the impairment while performing common manipulative tasks in order for her/him to understand and gain empathy with the impaired person. The developed tool is based on a wristattached desktop haptic interface with a workspace that is comparable to that of the arm of the user. Such device is able to exert controlled forces on the user's wrist and induce hand-tremor whose frequency and amplitude is correlated with different pathologies. The control of the device is based on the recording and playback of tremor signals acquired by a motion tracker. In this paper, we present the system with its dynamic characteristics and three different types of controller are experimentally tested and compared.
\end{abstract}

\section{INTRODUCTION}

In the field of Engineering Design, there are several products and services that are characterized by having a strong component of physical interaction with the end-user. Consumer electronic devices, furniture, office equipment, car interiors and computer-interfaces are just few examples of them. The design process for such products has to go through an in-depth analysis of the usability and acceptance by the end-users. In answer to this need, design practices have evolved toward a User Centered Approach and, very recently, the concept of empathy, borrowed from psychological science, has been introduced as a possible approach for the design of products and services. Empathy is the capability of recognizing emotions experienced by another subject and in the context of design it means to get an understanding of the end-user point of view. The discipline of Empathic Design (EmD) was born with the aim of proposing a design approach that strongly relies in a deep understanding of the end-user perspective and her/his relation with the products.

A very recent research branch of Engineering Design investigates the possibility of applying EmD principles to Inclusive Design [1], i.e. design systems that are accessible to all, including to special users such as the disabled or elderly. Actually, disabilities caused by pathological conditions or ageing can strongly influence the relationship between user and designed product. Traditional techniques for getting

This research is partially supported by the European project VERITASVirtual and Augmented Environments and Realistic User Interactions to achieve Embedded Accessibility DesignS (Project No. 247765), 7th Framework Programme, Theme FP7-ICT-2009.7.2, Accessible and Assistive ICT.

Authors are with PERCRO Laboratory of TeCIP Institute, Scuola Superiore Sant'Anna,Pisa, Italy.

Email: $\{$ g.rosatipapini, m.fontana $\} @$ sssup.it empathy with end-users are often hardly applicable in case of disabled users. This is because of the costs associated with the analysis done on large numbers of subjects for each of the many different types of end-user that should be involved.

A method for designer-empathizing with disabled endusers has been proposed and validated by Cardoso and Clarkson [2]. The basic idea is to equip the designer with passive hardware devices, called "capability-loss simulators", that limit the perceptual and motor capabilities of the designer in a way to temporarily simulate the end-user's reduced abilities.

Similar empirical studies have been conducted by several car manufacturers and lately at MIT within the project AGNES (Age Gain Now Empathy System) where researchers have developed an ageing suit able to introduce several mobility and vision impediments [3].

Such systems effectively simulate several visual/motor impairments associated with ageing. However, due to their passive nature, they lack flexibility and require long times to be donned, set up and tuned.

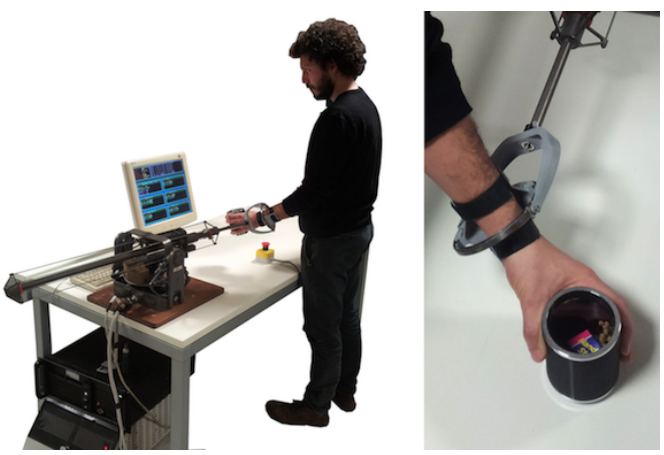

Fig. 1. Overall system employed for the generation of tremor composed by a haptic interface, controller and a novel end-effector spherical wrist.

Recently, researchers have started to investigate the possibility to simulate disabilities through computer-based programmable Human Computer Interaction (HCI) systems. The main goal is to develop more flexible tools that enable the simulation of several types of impairment with a level of severity that can be easily set by programming.

The simulation of disabilities has been studied for visual impairments in the field of web-design and word processor software with the development of simple software tools [4], [5]. A dedicated system for the design of accessible user interfaces based on EmD principles has been developed by Briner et al. [6]. 
In this context, within the research carried out in the European Project VERITAS, we consider a set of tools that are dedicated to the simulation of first person experience of a set of motor disabilities with more flexibility and with the possibility of rapidly changing system parameters [7].

In this paper, we present a system that aims at simulating the experience of manipulating/handling objects when affected by hand-tremor due to neurological diseases. Handtremor can be associated with several types of disabilities such as Parkinson, Essential Tremor, Cerebellar Tremor, etc.

In order to induce tremor on a healthy subject, we use a 3 Degree of Freedom (DoF) haptic interface called Grab [8]. The system, with its main components, is represented in Figure 1. The user stands in front of a table with the device attached to her/his forearm and is free to manipulate and explore objects on the table. In the final application, the device has been tested in collaboration with the house appliances company Indesit, focusing on the interaction with gas hobs in a real kitchen [9].

The paper is structured as follows. The second section of the paper briefly introduces the hand-tremor pathologies and qualitatively and quantitatively describes the kinematics of the associated movements. The third section presents the overall system employed to simulate hand-tremor and the experimental characterization of the system equipped with a novel end-effector. The fourth section shows the control strategies that have been designed to generate hand-tremor in the range of $4-12 \mathrm{~Hz}$ without interfering with the natural low-frequency movements of the arm. In the sixth section we show a preliminary experimental validation of the control obtained by recording, amplifying and playing back the natural tremor of a healthy subject.

\section{HAND-TREMOR SIMULATION}

\section{A. Hand-tremor}

Tremor is a common disturbance of movement, and it is defined as a rhythmic and oscillatory movement of a body part, caused by involuntary repetitive muscle contractions. It can vary in frequency and amplitude, and is influenced by motor, physiological, or psychological factors. A tremor is most often noticed in hands and arms, but it may affect several parts of the body. Tremor can be classified as:

- Resting: tremor occurs when muscles are resting. The tremor can disappear or become less noticeable when muscles move.

- Intention: tremor occurs at the end of a purposeful (intended) movement, such as writing, pressing a button, or reaching for an object. The tremor can disappear while the affected body part is at rest.

- Postural or actions: tremor occurs when holding the arm or leg against gravity or other forces in a position. This may happen when lifting a weight, or holding the arms out, or standing up straight.

Everyone has some tremor that superimposes on the voluntary movements of the limbs. Stress, fatigue, anger, fear, caffeine, and cigarettes may temporarily make this type of "natural" tremor increase.
However, there are several diseases whose symptoms consist in levels of tremor that influence and sometimes impede the normal execution of Activities of Daily Living (ADL).

The three most diffused types of pathologies associated with relevant levels of hand-tremor are: Essential tremor, Parkinson and Cerebellar Tremor. A comprehensive study and survey of all types of disease that are associated with tremor has been conducted by Wyne in [10]. A numerical investigation of the spectral analysis of tremor is reported in [11].

Each type of tremor has its own characteristics that varies according to the type of disease. However, the detailed characteristics of resting, intention and postural tremor may vary from patient to patient. Obviously, the simulation of all the aspects that are associated with tremor pathologies would be desirable but would require complex and expensive systems. In this work, we are focusing on postural tremor and its effects on manipulation/handling of objects. Thus, functionally speaking, we aim at developing a system able to induce in the user's hand a programmed vibration of a few millimetres (2-4 mm) in the frequency range of 4-12 Hz.

\section{B. Tremor induction strategies}

Few scientific works have been conducted on the simulation of a programmed hand-tremor. However, several researchers have investigated solutions for actively reducing hand-tremor. Active tremor compensation techniques have been studied as an aid for reducing hand-tremor for people affected by tremor diseases but also to improve human abilities during very accurate manipulation tasks (i.e. surgical operations) by cancelling the natural hand-tremor of healthy users.

Tremor compensation tools for enabling people affected by tremor disease to accomplish manipulative tasks have been studied in different research projects. Avizzano et al. [12] developed a system for enabling people affected by tremor associated with Multiple Sclerosis to interact with a PCbased environment.

In surgical applications, the reduction of physiological tremor is sought after in high precision manipulative actions. Active hand-held instruments able to actively compensate for hand-tremor have been developed in [13], [14].

In the first stage of our work, we analysed different strategies that could be adopted for mechanically producing this rhythmic movement. The possible identified strategies make use of three possible devices:

- Desktop haptic interface: the vibration movement can be induced by a desktop haptic interface programmed to impose a controlled force on the hand/arm of the user.

- Portable eccentric mass: the vibration is generated by a rotating eccentric mass that is mounted on the user's wrist.

- Electro-stimulation: the vibration can be generated by direct electrical stimulation of arm muscles. 


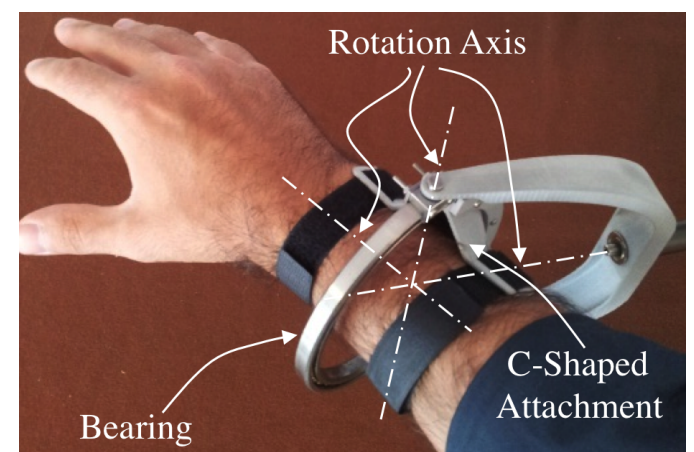

Fig. 2. Spherical joint implemented with rapid prototyping manufactured components and commercial Kaydon Reali-Slim ${ }^{\circledR}$ Bearing K10008CP0.

Electro-stimulation is potentially very promising in terms of fidelity of simulation, low weight and low power of the actuation system. Unfortunately, it shows several drawbacks such as annoying/painful stimulation and requires a lot of time to be set up. The portable eccentric mass solution could be doable, but due to the typical low-frequency of vibration $(4 \mathrm{~Hz})$ it would require large masses (in the range of $0.5-1 \mathrm{~kg}$ ) leading to excessive weight. The desktop haptic interface is a promising solution since the device is already designed for applying programmed forces on the human body. However, the device must guarantee the needed frequency-bandwidth/workspace and the controller has to be carefully designed in order not to interfere with natural voluntary movements. Having in mind the final application of the simulator, the solution employing the desktop haptic interface has been selected as the most suitable one.

\section{SYSTEM DESCRIPTION}

\section{A. Haptic interface}

The system that has been employed for the generation of artificial hand-tremor is the 3 Degrees of Freedom (DoFs) haptic interface developed by Scuola Superiore Sant'Anna, called Grab [8]. The Grab, is a serial kinematics desktop force-feedback device with a two rotary joints and one prismatic joint. It employs grounded motors and steel cabledriven transmissions. The design of the device was originally optimized for having low inertia, low friction and large isotropic workspace that is comparable to that of a human arm. The Grab is able to exert continuous forces in a bandwidth of $40 \mathrm{~Hz}$ over any desired direction with magnitude of $4 \mathrm{~N}$ in the worst condition (typically 6$7 \mathrm{~N}$ ). All DoFs are sensorized with high resolution angular optical encoders which guarantee an end-effector position resolution of $0.1 \mathrm{~mm}$. Stiffness measured at the end-effector is typically in the range of 6-7 N/mm. The end-effector of Grab was originally equipped with a small spherical joint that integrates a thimble for fingertip attachment or a sensorized plate that is employed when high accuracy is required [15].

For the purpose of hand-tremor simulation, the user needs to have his hands completely free for manipulating and exploring surfaces and objects. For this reason, we have designed a novel passive $3 \mathrm{DoF}$ spherical joint whose last link is tightly fasten around the user's forearm, close to the wrist, using velcro strips (see Figure 2). The joint is made of light-weight polyamide charged with aluminum powder, manufactured by Direct Printing rapid prototyping. The second of the three joints is implemented with a large diameter bearing (Kaydon Reali-Slim ${ }^{\circledR}$ Bearing K10008CP0) in order to place the center of the spherical joint on the axis of pronosupination motion of the forearm. An ergonomic c-shape attachment that envelops the forearm of the user has been design to have a comfortable and solid doublesupported connection. Such spherical joint has been designed aiming at maximizing stiffness and minimizing the mass, in order not to compromise the frequency response of the system. The final design of the joint presents a stiffness of $10 \mathrm{~N} / \mathrm{mm}$ (higher than that of the Grab haptic device) and a total weight of $190 \mathrm{~g}$.

\section{B. Complete setup}

The haptic interface is driven by a control unit that is equipped with encoder counters, Digital-to-Analog converters and current drivers. The controller runs at a refresh rate of $1 \mathrm{kHz}$ that is far more than the minimum required for controlling movements in the desired tremor-bandwidth (4$12 \mathrm{~Hz}$ ).

The complete system is composed by:

- the Grab haptic interface with the novel developed wrist attachment;

- a current-driver box that includes the driving electronics DC $48 \mathrm{~V} /-400 \mathrm{~W}$ power supply;

- a real-time operating system XPC target machine employed for implementation of the developed controllers.

The control is implemented using MATLAB/Simulink and loaded on the real-time target through Ethernet connections.

No force sensors are employed since the low friction/inertia of the mechanical structure and the type of employed motors allow reliable estimation of the exerted force directly from the readings of the motor currents.

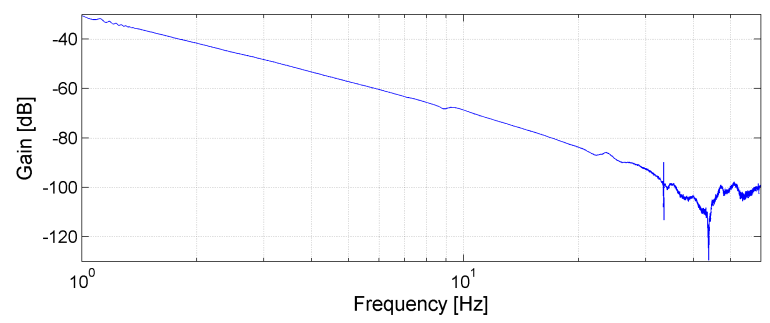

Fig. 3. Bode plot of the ratio between displacement over applied motor force when the end-effector is not attached to the arm of the user. Plot is obtained with $6 \mathrm{~N}$ amplitude chirp signal.

\section{System characterization}

As previously stated, the Grab haptic interface was originally designed to exert forces on the fingertip of the human hand. The system dynamics has been characterized in [16], however, in the present system, the attachment component has been modified introducing variation to mass properties. 
In this sub-section, we show the characterization of the dynamic properties of the overall system equipped with the novel end-effector. For the sake of clarity, we show results for only one DoF (the linear prismatic joint) of the device, that is the most critical in terms of bandwidth and maximum force performance. A Bode plot of the response of the system, in terms of the ratio between displacement of the end-effector and the equivalent end-effector force $x(f) / F(f)$, is shown in Figure 3, when the Grab is not attached to user's arm (noload condition). As can be observed, the plot represents the typical behaviour of a 2-mass system with an anti-resonant and resonant frequency in the range of $40-50 \mathrm{~Hz}$.

The dynamic response is modified when the end-effector is attached to the forearm of the user since the impedance of the arm reduces the amplitude introducing a highly damped double-pole zero system, with resonance and anti-resonance in the range of 3-6 Hz. The mechanical impedance of the user's arm is variable depending on the considered subject and the assumed posture. Figure 4 shows in solid line a plot of the amplitude of vibration of the forearm of the user that corresponds to a chirp signal with an intensity of $1 \mathrm{~N}$ in the frequency range of $1-15 \mathrm{~Hz}$. Measures are taken when the user is standing in front of the testing table with her/his hand in the center of the workspace, the arm disposed in a vertical plane and the elbow forming a right angle. In the same figure, the amplitude of the corresponding induced vibration of the fingertip is plotted. Fingertip vibration is acquired through the contact-less magnetic motion-tracker Polhemus Fastrak. The amplitude of the vibration in the range of interest (4$12 \mathrm{~Hz}$ ) varies from a maximum of $1.3 \mathrm{~mm} / \mathrm{N}$ to a minimum of $0.3 \mathrm{~mm} / \mathrm{N}$.

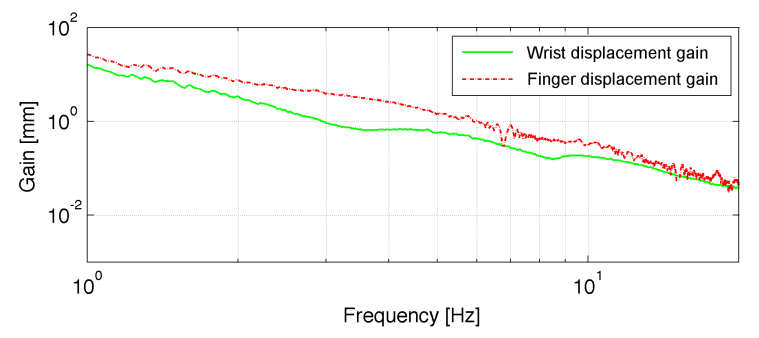

Fig. 4. Bode plot of the displacement of the end-effector of the haptic interface (solid line) and the displacement of the finger of the user (dotted line) in the range of 1-15 Hz. Displacements correspond to a force amplitude of $1 \mathrm{~N}$ and are measured with Polhemus Fastrak.

\section{TREMOR CONTROLLER}

The simulation of hand-tremor, with the described set up, can be implemented according to different strategies. The use of accelerometers, velocity sensors, and/or force sensors could be desirable, but in this study we decided to keep the complexity of the system as low as possible.

The simple designed control system is based on recording and playback strategy - i.e. recording hand trajectories of persons affected by tremor and playing them back through the developed system. The recording phase consists of measurements on patients, initially focussing on Parkinsonians, using a motion tracker (Polhemus Fastrak) which is able to acquire the 3D trajectories of small sensors attached to the user's wrist. The same trajectory is imposed on the user's wrist through properly controlled applied forces.

Thus, the acquired trajectories are employed to generate a reference control signal for the device. In order not to interfere with the voluntary movements of the user, the acquired trajectory $x_{r e f}(t)$ is filtered with a 2nd-order Chebyshev filter with normalized passband edge frequency from $3 \mathrm{~Hz}$ to $13 \mathrm{~Hz}$ and $0.1 \mathrm{~dB}$ of peak-to-peak ripple in the passband. The filtered trajectory $x_{r e f}^{*}(t)$ is employed in three different controllers: open-loop force control, band-pass position control and adaptive force-amplitude control.

It is important to underline that behind this control strategy a basic assumption is made that: if we impose to the distal part of the forearm of a healthy person the same trajectory of the forearm of a tremor-affected subject, the generated hand-tremor in the healthy subject is comparable to the handtremor of the disabled subject.

\section{A. Open-loop force controller}

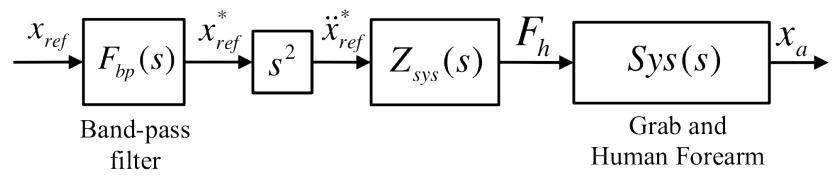

Fig. 5. Scheme of the open-loop controller. The commanded force $F_{h}$ is calculated as acceleration times the estimated impedance of the system measured in the center of the workspace $Z_{S Y S}$.

The simplest control strategy is in open-loop. Thus, the force that is imposed on the forearm of the user is proportional to the impedance of the arm multiplied by the acceleration computed by double time-derivation of $x_{r e f}^{*}(t)$ (see Figure 5). The impedance of the system is estimated by applying a chirp signal of $5 \mathrm{~N}$ in a range of $1-60 \mathrm{~Hz}$ in the center of the workspace when the user's forearm is attached to the device. The controller shows very low disturbances in the spectrum of low-frequency voluntary movements introducing minimal energy in the range of $0-4 \mathrm{~Hz}$. However, the amplitude control results are quite inaccurate since the system impedance is different from user to user and varies with the position in the workspace.

\section{B. Band-pass position controller}

The force delivered to the system is calculated on the basis of a traditional position control but the force signal is band filtered in order not to interfere with the low-frequency voluntary movement and to reduce high frequency noise. The recorded and filtered signal $x_{r e f}^{*}$ is employed as reference signal. The actual position is band-filtered with the same $F_{b p}(s)$, then it is subtracted from the reference signal and the result is fed back through a compensator $\left(C_{p}(s)\right)$. A scheme of the band-pass position controller is represented in Figure 6. 


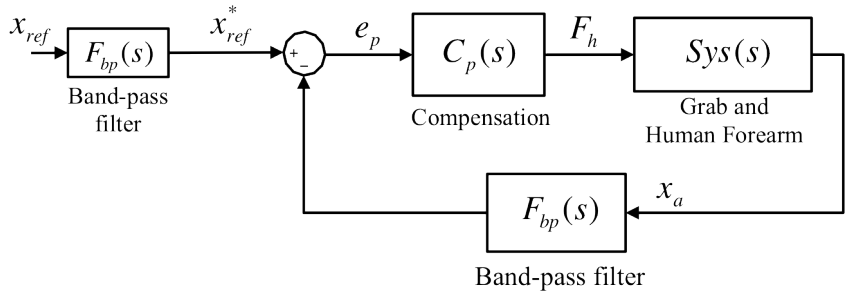

Fig. 6. Scheme of the band-pass position control. The commanded force $F_{h}$ is calculated through a compensation of the position error given by the difference between the filtered reference trajectory $x_{r e f}^{*}$ and the filtered actual position $x_{a}$.

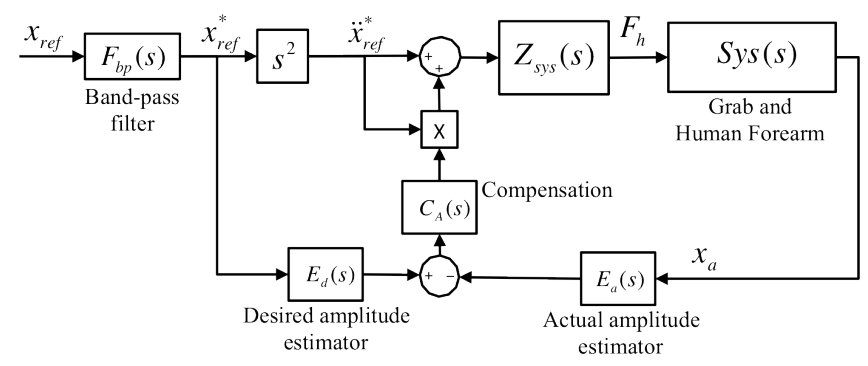

Fig. 7. Scheme of the adaptive force-amplitude control. The commanded force $F_{h}$ is calculated as in the open-loop controller but introducing a compensation calculated through estimating the amplitude error times the acceleration of the reference trajectory $\ddot{x}_{r e f}^{*}$.

\section{Adaptive force-amplitude controller}

Adaptive force-amplitude control is based on the previously presented open-loop force control but the force amplitude is compensated according to actual estimation of the amplitude of the imposed vibration (see Figure 7). The recorded and filtered tremor-signal $x_{r e f}^{*}$ is analysed and the desired amplitude $A_{d}$ is computed through an estimator based on a low-pass filter $E_{d}(s)$. Figure 8 shows a sample of $14 \mathrm{~s}$ of recorded tremor signal acquired on a healthy subject together with the estimated desired amplitude $A_{d}$. The actual amplitude $A_{a}$ is estimated from the encoder signal with a similar estimator based on a low-pass filter $E_{a}(s)$. The two values are subtracted generating an amplitude error that is fed back into the force loop through a compensator $C_{A}(s)$.

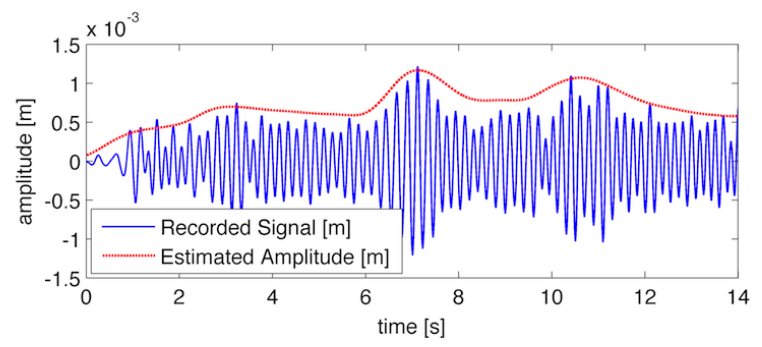

Fig. 8. Plot of the output of the amplitude estimator $A_{a}$ (dashed-red line) and recorded signal $x_{r e f}^{*}$ (solid-blue).

\section{EXPERIMENTAL TEST}

In this section, some preliminary results of functional tests are reported in order to assess the capability of the system to reproduce a recorded trajectory and to compare the different strategies of control. In this early stage of development, no patients have been involved in the experiments. The reference trajectory was acquired on a healthy subject and artificially amplified by a constant factor. The recorded and amplified signal has been employed as reference for the three controllers described in the previous sub-section (Section IV).

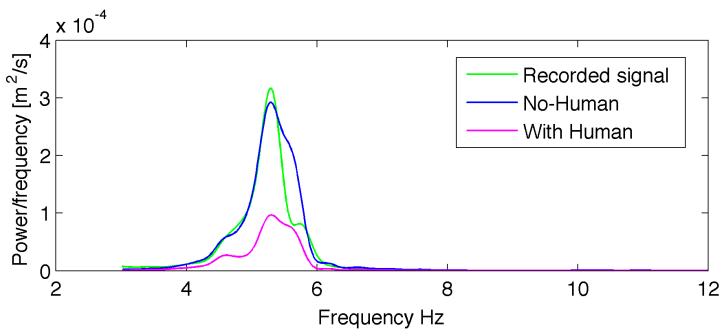

Fig. 9. Plot of the power spectrum for the open-loop controller described in Section IV-A. Comparison between the spectrum of the reference signal, output signal when the haptic interface is not connected (No-Human) and connected (With Human).

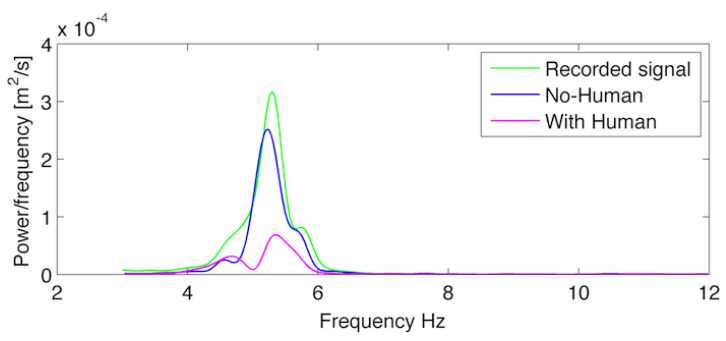

Fig. 10. Plot of the power spectrum for the band-pass position controller described in Section IV-B. Comparison between the spectrum of the reference signal, output signal when the haptic interface is not connected (No-Human) and connected (With Human).

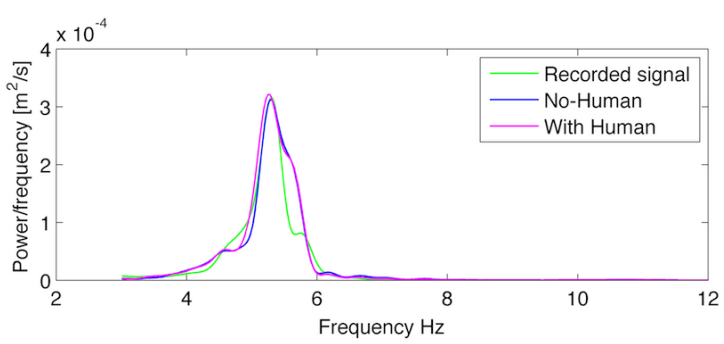

Fig. 11. Plot of the power spectrum for the adaptive force-amplitude controller described in Section IV-C. Comparison between the spectrum of the reference signal, output signal when the haptic interface is not connected (No-Human) and connected (With Human).

In order to compare the performance of the three types of controller, the plot of the velocity Power Spectrum (PS) are shown. In each of the Figures 9, 10 and 11 three PS plots are shown which refer to: (1) velocity PS of the recorded signal, (2) velocity PS of the response when the haptic interface is free, and (3) velocity PS of the response when the haptic interface is attached to the forearm of the user. It is interesting to notice that the plot of open-loop force controller 
(Section IV-a) shows a good matching between the spectrum of the induced vibration and the recorded signal when the haptic interface is free. But as soon as the device is attached to the forearm of the user the error becomes much larger (60\% of peak). The position band-pass controller (Section IV-B) shows minor differences between the spectrum of the recorded and induced tremor when the haptic interface is not connected. Also in this case there are major differences when the haptic interface is connected ( $70 \%$ of peak). The adaptive force-amplitude controller resulted the best solution, showing a good matching between the recorded and induced spectrum both in the case of free and attached haptic interface $(10 \%$ of peak).

\section{CONCLUSIONS}

Empathic Design (EmD) is an approach for the design of products that have a strong component of interaction with end-users. Recently, EmD has been applied in the field of accessible/inclusive design for products that are required to be accessible for all, including people with reduced abilities. In this context, we developed a tool for allowing designers to have a first person experience of the impairment of end-users who are affected by hand-tremor caused by diseases such as Parkinson or Essential Tremor. A desktop haptic interface with a large workspace has been employed to induce controlled vibrations on the hand of a healthy user to simulate the effect of hand-tremor disability when manipulating and handling objects.

A specially designed lightweight spherical joint has been studied for connecting the haptic device to the forearm and effectively transmitting the needed oscillating forces. The setup allows the user to interact with real objects while the haptic interface introduces vibratory movements that superimpose on the voluntary actions.

Three controllers for the haptic interface, which allow the user to program the level of induced tremor, have been studied. The controllers have been conceived and designed in order to minimize disturbances on low-frequency voluntary motions and to robustly control the amplitude of vibration of the user's wrist. The three controllers, which are based on different approaches, have been tested and compared with respect to their accuracy and robustness. The adaptive forceamplitude technique, that makes use of a force-based control compensated with an estimation of the vibration-amplitude error, resulted the best technique.

In the near future, the following activities are foreseen to be conducted: (1) a campaign of experimental acquisition on a set of patients affected by tremor diseases with the aim of acquiring trajectories to be employed as an input for the developed system; (2) a comparative analysis that aims at characterizing the correlation between tremor that is imposed to the user's wrist and the obtained tremor to the user's hand; (3) an in depth study on the correlation between imposed artificial tremor and resulting level functional impairment.

\section{REFERENCES}

[1] D. McDonagh, "Do it until it hurts! empathic design research," Design principles and practices: an international journal, vol. 2, no. 3, pp. 103-109, 2008.

[2] C. Cardoso and P. Clarkson, "Simulation in user-centred design: Helping designers to empathise with atypical users," Journal of Engineering Design, vol. 23, no. 1, pp. 1-22, 2012.

[3] "Agnes - age gain now empathy system." http://agelab.mit.edu/agnesage-gain-now-empathy-system.

[4] T. Oikonomou, K. Votis, D. Tzovaras, and P. Korn, "An open source tool for simulating a variety of vision impairments in developing swing applications," Universal Access in Human-Computer Interaction. Addressing Diversity, pp. 135-144, 2009.

[5] C. Strobbe, B. Frees, and J. Engelen, "An accessibility checker for libreoffice and openoffice. org writer," Computers Helping People with Special Needs, pp. 484-491, 2012.

[6] K. Breiner, T. Wchner, and M. Brunnlieb, "The disability-simulator: Simulating the influences of disabilities on the usability of graphical user interfaces," Lecture Notes in Computer Science, vol. 6779 LNCS, pp. 109-118, 2011.

[7] M. Fontana and M. Carrozzino, "VR interaction tools for motor impairment simulation," in Proc. of the IADIS Int. Conf. on Interfaces and Human Computer Interaction 2011, Multi Conf. on Computer Science and Information Systems, MCCSIS 2011, pp. 509-511, Jul. 2011.

[8] M. Bergamasco, F. Salsedo, M. Fontana, F. Tarri, C. Avizzano, A. Frisoli, E. Ruffaldi, and S. Marcheschi, "High performance haptic device for force rendering in textile exploration," Visual Computer, vol. 23, no. 4, pp. 247-256, 2007.

[9] M. Fontana, G. Rosati, R. Vertechy, M. Carrozzino, and M. Bergamasco, "Haptic hand-tremor simualtion for empathic design: Preliminary tests," in Proceedings of the IADIS International Conference Interfaces and Human Computer Interaction 2013, Multi Conference on Computer Science and Information Systems, MCCSIS 2013,, IEEE, Jul. 2007.

[10] K. Wyne, "A comprehensive review of tremor," Jaapa, vol. 18, no. 12, pp. 43-50, 2005.

[11] M. Gresty and D. Buckwell, "Spectral analysis of tremor: understanding the results.," Journal of Neurology, Neurosurgery \& Psychiatry, vol. 53 , no. 11 , pp. 976-981, 1990.

[12] C. Avizzano and M. Bergamasco, "Technological aids for the treatment of tremor," in Sixth International Conference on Rehabilitation Robotics (ICORR), 1999.

[13] C. N. Riviere, W. T. Ang, and P. K. Khosla, "Toward active tremor canceling in handheld microsurgical instruments," Robotics and $\mathrm{Au}$ tomation, IEEE Transactions on, vol. 19, no. 5, pp. 793-800, 2003.

[14] W. Latt, U.-X. Tan, C. Shee, and W. Ang, "A compact hand-held active physiological tremor compensation instrument," in Advanced Intelligent Mechatronics, 2009. AIM 2009. IEEE/ASME International Conference on, pp. 711-716, IEEE, Jul. 2009.

[15] M. Fontana, S. Marcheschi, F. Salsedo, and M. Bergamasco, "A three-axis force sensor for dual finger haptic interfaces," Sensors (Switzerland), vol. 12, no. 10, pp. 13598-13616, 2012.

[16] S. Marcheschi, F. Salsedo, M. Fontana, F. Tarri, O. Portillo-Rodriguez, and M. Bergamasco, "High performance explicit force control for finger interaction haptic interface," in Proceedings of the Second Joint EuroHaptics Conference and Symposium on Haptic Interfaces for Virtual Environment and Teleoperator Systems, WHC '07, pp. 464469, IEEE, Mar. 2007. 